BIBIANA DUARTE ABADÍA ${ }^{a}$

RUTGERD BOELENS ${ }^{\mathrm{b}}$

LUCAS DU PRÉc

\title{
ALIANZAS ANTIEMBALSE Y LUCHAS HIDROEPISTEMOLÓGICAS. LOS RETOS DE LA GESTIÓN COLECTIVA DEL AGUA EN EL RÍO GRANDE, MÁLAGA, ESPAÑA
}

\author{
RESUMEN
}

En este artículo analizamos las luchas epistemológicas y retos que ha tenido el movimiento social en la subcuenca del río Grande frente a los diversos proyectos modernizadores que intentan transferir el agua rural hacia la ciudad de Málaga. El primero asociado a la construcción de un gran embalse y el segundo con la modernización de los sistemas de riego tradicionales. Concluimos que el reto está en desenmascarar el régimen de verdad que respalda los proyectos de modernidad hidráulica, reivindicar los derechos de uso y control colectivos del agua y así generar procesos de apropiación comunitaria para la defensa del río como un bien socio-ambiental, económico-cultural y político-epistemológico.

Palabras clave: utopía hidráulica; gubernamentalidad; territorios hidrosociales; políticas hídricas; Málaga; España

\section{Abstract}

\section{ANTI-DAM ALLIANCES AND HIDROEPISTEMOLOGICAL STRUGGLES. THE CHALLENGES OF THE COLLECTIVE MANAGEMENT OF WATER IN THE RÍO GRANDE, MÁLAGA, SPAIN}

In this article, we analyze the water knowledge struggles and challenges that the Rio Grande sub-basin's social movement has faced when encountering the different modernistic projects that seek

a Centro de Estudios y Documentación Latinoamericanos (CEDLA), Universidad de Ámsterdam, Países Bajos. bibiana.duarte@gmail.com

b Department of Environmental Sciences, Wageningen University, The Netherlands. rutgerd.boelens@wur.nl CEDLA Centre for Latin American Research and Documentation, The Netherlands.

Department of Geography, Planning and International Development Studies, University of Amsterdam, The Netherlands.

c Departamento de Ciencias Ambientales, Universidad de Wageningen, Países Bajos. lucasdupre23@gmail.com Fecha de recepción: octubre 2018. Fecha de aceptación: diciembre 2018. 
to transfer rural water to Malaga city. The first relates to a large dam construction, the second to the modernization of traditional irrigation systems. We conclude that the movement's challenges are to unpack the truth regimes that support these hydraulic-modernistic projects, to claim for collective water use and control, and to engage in a bottom-up «commoning» process for defending the river's socio-environmental values, cultural-economic importance, and epistemological meaning and existence.

KeYwords: hydraulic utopia; governmentality; hydrosocial territories; water politics; Málaga; Spain

\section{INTRODUCCIÓN}

La canalización, el almacenamiento y el control de los flujos de agua para responder a las demandas de riego, de hidroelectricidad, de consumo doméstico, de producción industrial y de protección contra las inundaciones, sobre todo en las ciudades, se han hecho posible a través de la construcción de presas. Estas obras han marcado procesos históricos en la modernidad, convirtiéndose en iconos de progreso, modernidad y nacionalismo (Nixon, 2010, Kaika, 2006; Swyngedouw, 1999, 2007; Swyngedouw y Boelens, 2018). También han determinado nuevas relaciones entre la vida urbana, rural y natural, caracterizados por la dependencia del mundo urbano respecto al mundo natural/rural (Delgado y Del Moral, 2016; Hommes y Boelens, 2017, 2018). Kaika (2006) sostiene que la modernidad ha logrado domar, controlar y disciplinar el orden natural por medio de la ciencia y la ingeniería, proceso indispensable para el desarrollo y crecimiento de las ciudades y que también ha sido conceptualizado por muchos académicos como la «urbanización de la naturaleza» (Ibid., 2006: 276).

En los últimos treinta años la subcuenca del río Grande, en la provincia de Málaga (Andalucía), ha sido objeto de diversos planes modernizadores que amenazan reordenar sus flujos de agua, los territorios y las poblaciones. En gran parte es la consecuencia del continuo crecimiento de la población, la industria y el turismo en la ciudad de Málaga. Específicamente, analizamos dos proyectos de modernización hidráulica que buscan transferir aguas rurales hacia la ciudad. El primero tiene que ver con la construcción de la presa de Cerro Blanco, incluida ya en el Plan Hidrológico de la Cuenca del Sur (PHCS) de 1995, y el segundo está basado en la tecnificación y modernización de los sistemas de regadíos tradicionales. Por medio de estos dos proyectos, ilustramos el papel y los retos que tiene el movimiento social de la subcuenca del río Grande para combatir los proyectos de la modernidad hidráulica capitalista que amenazan con debilitar la autogestión local y colectiva de las aguas que históricamente han contribuido a la reproducción cultural de este territorio.

En el transcurso de estos proyectos modernizadores se constituyen arenas políticas, en donde actores con intereses divergentes emplean estrategias, recursos y discursos distintos para conseguir sus objetivos, lo que se manifiesta en la contraposición y legitimación/deslegitimación de distintos sistemas de saber o repertorios de conocimiento. Este artículo analiza históricamente las diferentes fases por las cuales los diversos sistemas de conocimiento se 
enfrentan. Ilustramos la capacidad que tiene la movilización social para construir redes sociales con diversos actores y romper discursos modernizadores dominantes que amparan la construcción de las presas (véase también Duarte-Abadía y Boelens, 2019; Jiménez Sánchez y Poma, 2011; Poma y Gravante, 2015; du Pré, 2017).

Aplicando un enfoque de ecología política, el artículo expone los resultados de diversos períodos de trabajo de campo durante los años 2016-2017. En este periodo, se realizaron 83 entrevistas a diferentes escalas de investigación en la subcuenca del río Grande. La primera escala comprende las fincas, en donde participaron agricultores y la organización de regantes que hacen parte de las siete acequias que componen el sistema de riego de río Grande. La segunda escala abarca a los líderes sociales que conforman la «Coordinadora en Defensa del río Grande», movimiento social local principalmente representado por los habitantes del municipio de Coín opuestos a la construcción de la presa. La tercera escala se manifiesta por la participación de los académicos que apoyaron el movimiento social; y en la cuarta escala se entrevistaron a oficiales de la Empresa Municipal Aguas de Málaga (EMASA) y entidades encargadas de coordinar asuntos del agua, dependientes de la Consejería de Medio Ambiente en la provincia de Málaga. Las entrevistas se acompañaron de visitas de campo y revisión de bibliografía secundaria.

En la siguiente sección conceptualizamos como las luchas entre los diversos sistemas de conocimiento son producto de una construcción social que constantemente es moldeado por experiencias y confrontaciones entre los distintos «mundos de vida» — lifeworlds (Long, 2003). En la tercera parte, ilustramos las políticas urbano-modernizadoras y sus proyectos basados en los procesos de transferencia de agua desde lo rural hacia lo urbano. Para ello primero mostramos las diferentes fases por las cuales se plantea la construcción de la presa y sus respectivas contestaciones sociales que la rechazan. Luego, explicamos las paradojas que debe enfrentar el movimiento antiembalse y los moradores que defienden el río, especialmente la comunidad de regantes, frente a la entrada de intervenciones de modernización del sistema de riego tradicional del río Grande. Para ambos proyectos, la construcción de la presa y la instalación de riegos por goteo, analizamos como se enfrenta el conocimiento de los expertos (ingenieros) al conocimiento popular, sus respectivas alianzas, y los poderes que se constituyen para desvirtuar/imponer verdades y así transformar realidades. También examinamos cómo han surgido sistemas de conocimiento alternativos, basados en una revalorización de la historia, ecología y los apoyos socio-económicos locales de la subcuenca. En la última parte explicamos y concluimos como la lucha unificada para evitar la construcción de esta presa se complica, fragmentándose y debilitándose por la inserción de mecanismos menos visibles pero que se internalizan en las prácticas y conocimientos de los usuarios del río Grande, las comunidades de regantes. Es el caso de las políticas de modernización/tecnificación de los sistemas de riego tradicionales. 


\section{Modernidad hidRÁUlica, LUCHAS EPISTEMOlóGicas}

Y EL PAPEL DE LOS MOVIMIENTOS SOCIALES

En España, al final del siglo XIX, la construcción de grandes obras hidráulicas para interconectar y hacer buen uso de las aguas del país, emergió como la solución para revivir el desarrollo económico nacional. Corrientes ideológicas como el «Regeneracionismo» promovían una importante «política hidráulica». Así, por ejemplo, el líder regeneracionista Joaquín Costa (1911) glorificaba el riego campesino tradicional y la ampliación de los sistemas de regadío, pero mediante la construcción de embalses para aprovechar al máximo todos los flujos hídricos (Boelens y Post Uiterweer, 2013; Del Moral y Saurí, 1999; Duarte-Abadía y Boelens, 2019). Con la construcción de las presas se demostraría «progreso», «independencia»y «modernidad» (Gajic, 2007).

Los procesos de modernidad además de transformar, apropiarse e instrumentalizar la naturaleza y los ríos, también han generado nuevas estructuras sociales y de poder basadas en el dominio de ciertos conocimientos técnico-políticos. Es bien conocido como, en especial durante la época del Franquismo, la modernización hidráulica, de manera forzosa, se convierte en un principal motor de transformación socio-territorial, en el que paradójicamente la imposición de la razón se antecede de actos irracionales, prácticas violentas y mecanismos de exclusión que niegan otras formas de conocimiento y prácticas culturales (Fernández Clemente, 2000; Ortí, 1984; Swyngedouw, 2007).

El desarrollo tecnológico que ha acompañado el transcurso de la política hidráulica española, incluyendo las épocas de los últimos gobiernos de Zapatero y Rajoy, se ha caracterizado por incentivar diversas caras de modernización. Si bien la construcción de grandes obras hidráulicas de regular los ríos se ha reducido en comparación a lo que se registraba en el periodo de Franco, las nuevas políticas promueven desarrollos tecnológicos orientados a la modernización de los sistemas de riego tradicionales — con una visión claramente tecnicista-economicista- y la construcción de mega-proyectos en torno a las plantas desalinizadoras (Swyngedouw, 2015).

En la actualidad, más que imponer abiertamente, el proyecto de la modernidad utiliza mecanismos inclusivos y participativos para poder controlar lo diferente. Por ejemplo, es muy común observar como todos los procesos de tecnificación y modernización de sistemas de riego se esfuerzan por involucrar a las comunidades de regantes en las racionalidades de los grupos gobernantes. De esta manera se promocionan esquemas para el uso eficiente del agua, pero que en la práctica están conllevando a agudizar la distribución desigual y su extracción insostenible, afectando y apropiándose de los medios de vida de las poblaciones rurales (véanse también Duarte-Abadía et al., 2015; Duarte-Abadía y Boelens, 2016).

Tal como en la subcuenca del río Grande, las evidencias en las diversas culturas hídricas alrededor del mundo muestran que el continuo avance de una modernidad hidráulica universalista y capitalista se sostiene a costas de las realidades vernaculares, es decir de aque- 
llos dominios que aún mantienen cierta independencia de las fuerzas y leyes del mercado (Boelens, 2015; Delgado y Del Moral, 2016; Hommes y Boelens, 2017). A pesar de ello, muchos valores vernaculares, enraizados en diversas prácticas culturales —como por ejemplo los sistemas de riego «a manta» y sus usos, costumbres, reglas y prácticas consuetudinarios en la zona de Coín - han logrado coexistir con las transformaciones modernizadoras; a menudo son valores y conocimientos que están ocultos pero que son intrínsecos en las diversas estrategias de reproducción cultural y en la permanencia de las alteridades, de aquello que sigue siendo diferente y distinto (Illich, 1980). Este tipo de sabiduría está referida a los conocimientos del campesinado; es un conocimiento que constantemente se recrea a partir de la experiencia y desde las capacidades que tienen los agricultores/campesinos para coordinar y adaptarse a un escenario amplio de incertidumbres ecológicas y de factores socio-técnicos de tal manera que les permita obtener los resultados esperados para su subsistencia (Stuiver, Leeuwis y Van der Ploeg, 2004). Lejos de una representación romantizada, es un sistema de conocimientos de carácter holístico y que al estar atado a la práctica es creativo y dinámico, es decir que constantemente se construye como resultado de incorporar selectivamente ideas y experiencias previas, creencias, sentimientos, entre otros (Arce y Long, 1987). Y por ello mismo, las ciencias tecno-positivistas, formalmente acreditadas por los centros de conocimiento hidro-tecnológico dominantes, lo categoriza como conocimiento informal, atrasado e ineficiente.

Foucault (1980) conceptualiza estos conocimientos como subjugated knowledges, refiriéndose a estos como saberes que han sido descalificados por ser insuficientemente elaborados dentro de marcos conceptuales científicamente aceptados. Son formas de saber distintos al «poder disciplinante»; este último busca clasificar, normalizar, homogeneizar, y centralizar el conocimiento para con ello controlar la sociedad. Por lo tanto, la jerarquización de las formas de conocimiento que está implícita en el proceso social y se basa en relaciones de poder, autoridad y legitimidad (Long, 2003), constituye relaciones entre poder/conocimiento que emergen en procesos de interacción social. El encuentro de conocimientos dominantes y subalternos implica luchas entre actores y sus sistemas epistemológicos respectivos, arenas en que el uno busca controlar y dominar al otro mediante proyectos técnico-políticos y cultural-normativos, para que acepten marcos de significados particulares que posicionan sus formas de ver la vida y el comportamiento.

En este artículo analizaremos cómo los saberes vernaculares de los pobladores y activistas del movimiento social de río Grande responden activamente a la imposición de conocimientos técnico-científicos que buscan alterar los patrones de distribución y gobernanza del agua. Es una respuesta que está asociada a las contestaciones de otros movimientos sociales en España; reclaman que los procesos de modernización han conllevado a aumentar nuevas demandas hídricas sin resolver el fondo del problema hídrico. A nivel nacional se destaca la consolidación de la Nueva Cultura del Agua en 1995, un movimiento de académicos y activistas que junto con grupos ecologistas buscan replantear las políticas de gestión del agua; 
cuestionando, por ejemplo, el Plan Hidrológico Nacional en las últimas décadas (Copitzy, 2012). Este movimiento comenzó a proliferarse en distintas regiones de España y en el Valle del río Grande se manifestó primero por medio de la creación de la Plataforma Antipresa de Cerro Blanco y luego a través de la constitución de la Coordinadora de la Defensa del río Grande.

Las acciones de la Coordinadora, de manera general, no se centran en el rechazo genérico a la modernidad, sino que tienden a incluirla de manera crítica y reflexiva, como parte de sus proyectos y propuestas para sostener sus medios de vida y defender sus derechos de agua y del territorio (ver también Bebbington, 1996; Boelens, 2015). Así, las diferentes manifestaciones de la modernidad hidráulica, con sus respectivos avances tecnológicos, también están sujetas a ser contestadas, apropiadas, modificadas y alteradas, según las exigencias e intereses de las poblaciones directamente afectadas (Pfaffenberger, 1992). La siguiente sección examina las políticas/proyectos urbano-modernizadores que buscan apropiarse del control y acceso de las aguas de río Grande, las diversas respuestas sociales que se levantan para evitar que pase esto, así como los problemas y paradojas que enfrenta la movilización social cuando se ve confrontada con los proyectos que buscan modernizar los sistemas de riego tradicional.

\section{LAS POLÍTICAS URBANO-MODERNIZADORAS}

El modelo vertical de la política hidráulica franquista ha persistido durante buena parte de la etapa democrática, un periodo en el que la presión sobre las fuentes hídricas ha aumentado considerablemente (Estevan, 2008; Fernández Clemente, 2000). Sampedro y Del Moral (2014) analizan como este tránsito que vivió España hacia la modernidad hidráulica llevó a aumentar las demandas hídricas y a generar situaciones de escasez, causadas por la sobreexplotación de las fuentes subterráneas y superficiales. Estos autores sostienen que después de 1981 en Andalucía se construyeron más de 80 embalses, aumentando la oferta a los 11.000 $\mathrm{hm}^{3}$ en comparación a los $6.500 \mathrm{hm}^{3}$ existentes para 1987 (Sampedro y Del Moral, 2014: 41). En Málaga, al inicio de los años 2000, hubo un período de gran escasez hídrica que ha sido usado para legitimar la construcción de nuevos embalses. Sin embargo, a pesar del aumento de la disponibilidad del recurso hídrico embalsado en la década posterior, se seguía prolongando el decreto de sequía por muchos años (Estevan, 2008), para justificar la construcción de una presa sobre río Grande.

El discurso modernizador dominante de la escasez hídrica ha legitimado la construcción de grandes embalses en el siglo pasado, pero en las últimas décadas y en el presente siglo tiene un nuevo matiz: el ahorro y uso eficiente del agua bajo la introducción de tecnología de riego por goteo (Sanchis-Ibor et al., 2017). Esta tecnología se ha convertido en sinónimo exclusivo del término modernización, y sobre este ha construido un discurso, que de forma reiterada y explícita ha acusado al regadío tradicional de ser «ineficiente» (López-Gunn et al., 2012; Sanchis-Ibor et al., 2016, 2017). Sin embargo, esta misma tecnología se ha uti- 
lizado masivamente en Andalucía para crear nuevos regadíos sobre los secanos olivareros, conduciendo a la sobreexplotación de numerosos acuíferos (Corominas y Cuevas-Navas, 2017). La presurización del riego también se ha empleado como estrategia para liberar (hipotéticamente) recursos y satisfacer las crecientes demandas del sector turístico-residencial, las cuales también han justificado el desarrollo de nuevos proyectos de regulación fluvial, en ocasiones claramente sobredimensionados. Esta es la situación que se vive en la subcuenca de río Grande y que será descrita en la presente sección.

\subsection{Proyectos para embalsar y transferir las aguas de río Grande y movilizaciones sociales}

La construcción de la presa de río Grande está contemplada desde mediados de 1980 y su proyección se hace visible en el Plan Hidrológico de la Cuenca del Sur (PHCS) de 1995 en donde se le denomina la presa de Cerro Blanco. Esta se localizaría en los términos municipales de Guaro y Coín. La capacidad del embalse contemplaba $32 \mathrm{hm}^{3}$, con una superficie inundada de 240 ha y una capacidad de regulación de $25 \mathrm{hm}^{3}$ /año (Puche, 2003). Esta obra se justificaba desde la necesidad de regular las considerables avenidas del agua en el invierno. Informes oficiales y la prensa malagueña reclamaban: «río Grande tira al mar $80 \mathrm{hm}^{3}$ de agua al año y Málaga la necesita», y «Las grandes avenidas inundan Málaga y hay que regular el río» (Puche, 2003).

La presa inundaría 171 ha que comprendía las huertas y los regadíos de los pobladores del municipio de Guaro. La realización de esta construcción tenía como fin regular las transferencias de aguas de ríos vecinos, Guadiaro y Genal, para abastecer las demandas del oeste de la ciudad de Málaga (Jiménez y Poma, 2011; Gómez, 1998). Estos hechos, suscitaron la movilización de los agricultores de Guaro quienes se organizaron en el 2000 a través de la constitución de la Plataforma Antipresa de Cerro Blanco. Su lucha se centró en defender las huertas y la cultura de regadío tradicional árabe que todavía se mantiene vivo en el valle de río Grande. El arraigo de los agricultores de Guaro a sus huertas y acequias tradicionales, especialmente representado por la población mayor, se transmitió a las generaciones más jóvenes y se hizo sentir en las entidades oficiales locales, las cuales apoyaron las protestas de estos y lograron exponer sus posiciones en las instancias políticas regionales y frenar la construcción de esta presa en el 2001.

Desde lo local, la Plataforma Antipresa de Cerro Blanco recibió el apoyo de ambientalistas y activistas de Coín integrados en la Asociación Jara; a nivel regional miembros de Ecologistas en Acción ${ }^{1}$ participaron en la movilización, también lo hizo Grupo de Trabajo Valle de Genal quienes se opusieron a la construcción de la presa del Genal. ${ }^{2}$ Desde el ámbito nacio-

1 Ecologistas en Acción es una confederación de más de 300 grupos ecologistas de toda España, unificados en diciembre de 1998.

2 La presa del Genal se ubicaba en la provincia de Málaga, en la comarca de la Serranía de Ronda, donde nacen las aguas del río Guadalteba que entrega aguas al río Guadalhorce. 
nal, la Fundación Nueva Cultura del Agua (FNCA) fortaleció el debate a través de congresos y foros que se realizaron en los pueblos a ser afectados. De igual manera, la movilización se expandía adhiriéndose a otras contestaciones como la Plataforma en Defensa del Ebro y la Coordinadora de Afectados de Grandes Embalses y Trasvases. Ambas se oponían a las transferencias de agua de la cuenca del Ebro hacia el Mediterráneo (COAGRET) (Gómez, 1998; Puche, 2003). Estos movimientos se resistían a seguir asumiendo las consecuencias del deterioro de los ecosistemas acuáticos y los desplazamientos sociales derivadas de la anterior política hidráulica. Sus movilizaciones proclamaban acciones para proteger los pocos «ríos vivos», que quedaban en España y que se veían amenazados por la construcción de 120 nuevas presas y proyectos de trasvases de aguas. Aparentemente, la movilización multinivel tuvo efecto y la obra fue revocada.

Sin embargo, dos años después, en junio del 2006, el proyecto renació y fue aprobado por el Ministerio de Medio Ambiente: esta vez, en lugar de anunciar la construcción de una presa, hace referencia a la construcción de un azud, una presa de menor tamaño cuya función es derivar agua a través de la elevación de sus niveles (tendría $7 \mathrm{~m}$ de altura y $8,4 \mathrm{hm}^{3}$ de agua embalsada). Por lo tanto, el impacto de inundar 171 ha (8 \% del término municipal de Guaro), se sustituía por transferencia directa de agua hacia Málaga (Esquema 1).

Se construiría un canal de conducción de 38 kilómetros, con un tubo de 1,60 m de diámetro que tendría una capacidad de transportar $20 \mathrm{hm}^{3}$ anuales, $4 \mathrm{~m}^{3} / \mathrm{s}$, aguas que provendrían de las crecientes del río durante la época de invierno. Para los miembros de la FNCA, la ejecución de la obra significaba la conducción entera del río, porque de acuerdo a las dimensiones del diámetro del tubo de derivación, la capacidad plena del funcionamiento podía alcanzar los $126 \mathrm{hm}^{3} /$ año, es decir un volumen mayor de las aportaciones del río — según sus cálculos la aportación media extrapolada era de $80 \mathrm{hm}^{3}$ /año (ver Puche, 2003).

En el mismo año en que se aprobó el proyecto, 2006, comenzaron a llegar las cartas de expropiación para los pobladores que tenían tierras en río Grande. Alrededor de 25.000 personas serían afectadas por la implementación del proyecto, más 2.500 ha de regadíos que se perderían. ${ }^{3}$ Se vivía una situación de miedo e incertidumbre por parte de toda la población de Coín y Guaro, quienes en primera instancia acudieron a los grupos ecologistas locales, «La Asociación Cultural Jara». Así mismo convergieron otros miembros que habían sido parte de la Plataforma Antipresa de Cerro Blanco para entre todos conformar en septiembre del 2006 la «Coordinadora en Defensa del río Grande», de ahora en adelante Coordinadora. De esta manera, se unieron también los pueblos de Pizarra, Cartama, Alora, con un logo propio como símbolo de su lucha: «... hicimos unión del pueblo para poder salvarlo y en analogía pusimos un corazón y el rio yendo alli» (junio 22, 2015 Salvador Sánchez, presidente Asociación Jara).

3 Información obtenida a través de la Coordinadora de la Defensa del río Grande. 
La primera estrategia por parte de la Coordinadora fue conectarse con otros colectivos locales, entidades activistas, expertos y académicos pertenecientes a la FNCA. Durante este proceso de coalición, la Coordinadora inició una campaña informativa para los pobladores de río Grande y ciudadanos vecinos. Buscaron la documentación del proyecto, la estudiaron y analizaron bajo el apoyo intelectual de la FNCA, para luego transmitirla y divulgarla. Esta fue su segunda estrategia para afianzar el control sobre sus aguas y la defensa de sus derechos sobre el río: dominar el conocimiento integral del proyecto, asociándolo a las relaciones urbano-rurales en cuanto a oferta y demanda hídrica. De esta manera, se iban organizando los datos, montando los estudios y alegaciones jurídicas que argumentarían la inviabilidad de la presa y otras alternativas para el abastecimiento hídrico de Málaga.

La tercera estrategia de la Coordinadora consistió en mantener la independencia política durante su movilización. Se abstuvieron de recibir el apoyo de cualquier partido y así mismo no dejaron que ninguno de estos tomara la voz durante el proceso de movilización, de esta forma consiguieron articulación y credibilidad en la ciudadanía. La Coordinadora, durante su movilización, se dio cuenta que detrás de la construcción de la presa existían intereses políticos de captar subvenciones de la Unión Europea para la construcción de proyectos. La postura no partidista de la Coordinadora, junto con su creciente movilización en 2006 2007, incidió en el cambio de postura de los políticos locales, al coincidir con las elecciones municipales del 2007. Los candidatos se vieron comprometidos a rechazar públicamente el proyecto para no perder votos.

La cuarta estrategia de la Coordinadora, apoyada financieramente por la FNCA, fue realizar un estudio sobre la evaluación y valoración del estado ecológico de las aguas de río Grande, para demostrar frente al Ministerio de Medio Ambiente el alto grado de conservación de la biodiversidad y calidad de agua que tiene esta subcuenca en su parte alta. De esta manera, la Coordinadora buscaba que esta zona se declarará como Lugar de Interés Comunitario (LIC) ${ }^{4}$ y así evitar por la vía legal un nuevo intento de construcción de la presa. Sin embargo, en el 2009 el Ministerio de Medio Ambiente les solicitó pagar un estudio independiente, que estuviese avalado por una Universidad para hacer efectiva la consideración. La Coordinadora acudió a la Universidad de Málaga, pero por el alto costo, la Asociación Jara se vio obligada abandonar la vía legal para proteger el río Grande.

No obstante, en mayo del 2007 se descartó la construcción del Azud en el río Grande y en diciembre se anunció la obra que la reemplazaría. Se trataría de una conducción de $21 \mathrm{~km}$ que uniría el azud de Aljaima (ubicado en el cauce del río Guadalhorce, poco después de la desembocadura del río Grande) hasta la planta desaladora del Atabal, ubicada en la cabecera de Málaga. Esta alternativa desde un inicio había sido planteada por la Coordinadora. Así lo expone Salvador Sánchez (Junio 22, 2015): «Ofrecíamos alternativas... que Málaga

4 LIC es parte de la RED Natura 2000, las áreas de conservación de la biodiversidad de la Unión Europea. Son considerados espacios del territorio nacional que contribuyen a mantener o establecer el estado de conservación para favorecer hábitats naturales donde se encuentran especies de interés comunitario. 
tome el agua en cola del rio y no en cabecera, allí ya existía un azud desde hace mucho». Por su parte, uno de los expertos hídricos y miembro de la FNCA expone: «De allí se aprovechan el $80 \%$ de las aguas que se iban a sacar de la cabecera del río. Esa obra generó que no tuviera impacto en el cauce del rio, y se consiguió el mismo objetivo que la gran presa» (entrevista Marzo 23, 2017).

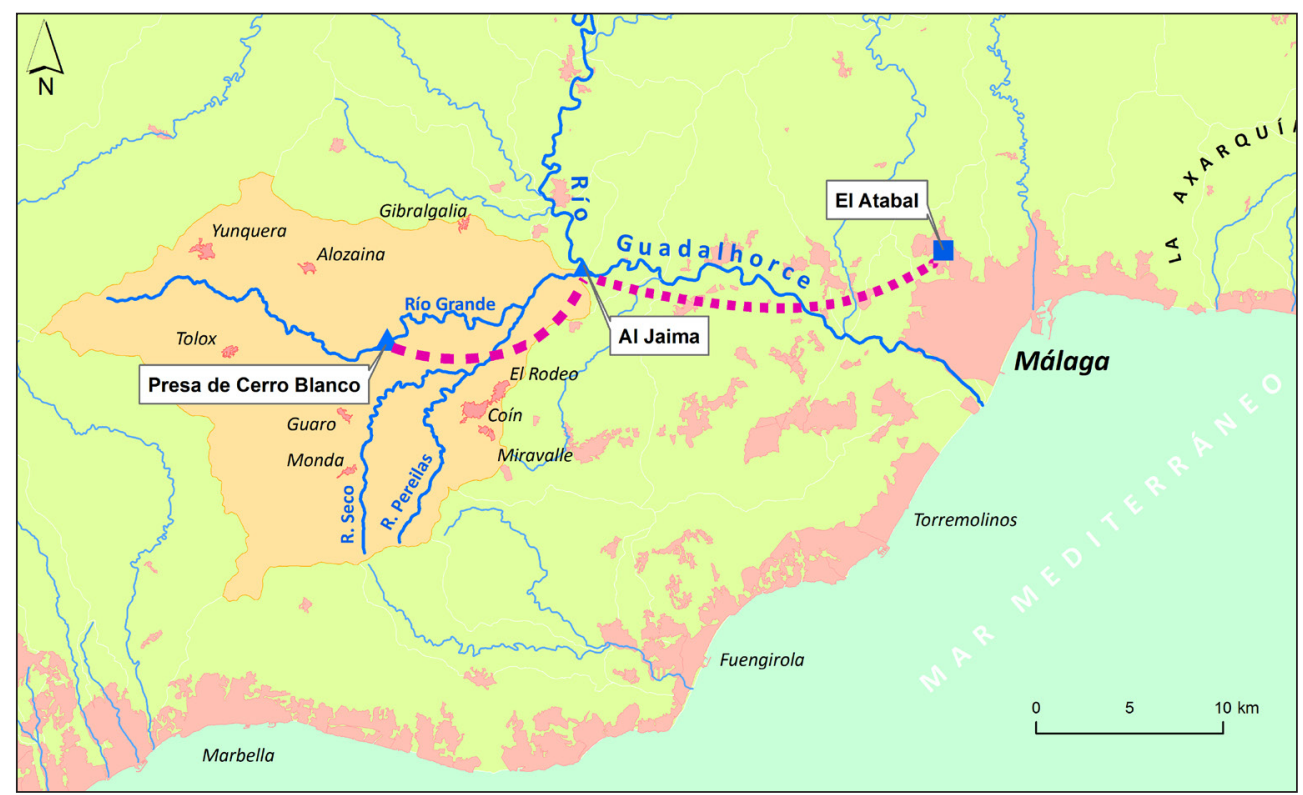

Figura 1. Esquema de las posibles obras hidráulicas en Río Grande. A la izquierda, la posible construcción de la presa de Cerro Blanco, también lugar del Azud con el tubo propuesto (38 km): el posible canal de conducción para transferir aguas rurales hacia Málaga. Sobre la derecha, la obra definitiva, canal de conducción de $21 \mathrm{~km}$ que une el Azud de Al Jaima con la planta desaladora de El Atabal.

\subsection{La modernización del sistema de riego tradicional}

La lucha por la defensa del río Grande vivo conllevó una movilización unificada y empoderada para evitar la construcción de canales y azudes que buscaban transferir las aguas rurales de Coín a la ciudad de Málaga. Paradójicamente, la ideología que motivó la movilización en cuanto a mantener el río Grande vivo, se convierte en el discurso que usan otros grupos ecologistas, que viven fuera del territorio, muchos dedicados a la pesca deportiva y con cercanas relaciones a la Junta de Andalucía, para cambiar las prácticas de aprovechamiento del 
río por parte de los regantes. Así lo expresaba Pepe Cortés, el aguador de la acequia de Guaro (Marzo 3, 2017): «Aquí el problema era llevarse el agua y desde entonces, como se echó para atrás, problemas son los que hemos tenido, porque el lema nuestro era "Río Grande Vivo", tal y cual... ¿Qué queréis vivo? ...pues ahora no vais a coger el agua». Durante, los veranos del 2009-2010 la comunidad de regantes de río Grande fue denunciada por la Fiscalía de Medio Ambiente. La demanda la presentó la Asociación para la Conservación Piscícola y de los Sistemas Acuáticos del Sur (ACPES).

Según estos ecologistas, la construcción de las tradicionales azudes que hacían los regantes para capturar las aguas del río e introducirlas por las acequias estaba ocasionando una masiva mortandad de peces, perjudicando el equilibrio de los sistemas naturales. Así lo había anunciado un socio de ACPES:" «Advertimos desde el año 2005 que hay un par de abusos por el río... Las causantes son las extracciones de las comunidades de regantes... Hicimos cada año denuncias y no hicieron nada. El interés del ACPES es el medio ambiente» (Antonio Pardo, socio de ACPES, mayo 18, 2017)

Para ACPES las causas de la sequía del río Grande en el verano están dadas por las múltiples extracciones de agua que se hacen del río, muchas de ellas ilegales (es decir, informales) y por ello había que comenzar a controlar las más visibles. En consecuencia, durante los veranos del 2009-2010, los servicios de guardia fluvial de la Agencia Andaluza del Agua iniciaron una vigilancia permanente sobre las prácticas de riego que ejercían las comunidades de regantes. Para los regantes estos procedimientos significaron un instrumento jurídico utilizado en su contra frente a la Junta de Andalucía. Sus prácticas consuetudinarias y derechos de agua se deslegitimizaban por no ser adecuadas con la protección del río.

Esta situación se agudizó más bajo el marco legislativo impuesto por la Directiva del Marco del Agua (DMA) de la EU. Introducía la necesidad de adoptar las medidas necesarias para proteger los ecosistemas acuáticos y recuperar el buen estado ecológico del río. De esta manera, se justificaba la modernización de regadíos, especialmente la sustitución de los riegos tradicionales (riego por gravedad o «a manta») por riego por goteo. Se concebía como una estrategia regulatoria para evitar la escasez hídrica y optimizar la redistribución del recurso en los demás sectores sociales. «Ellos tendrían que acabar con el riego a manta, porque es un despilfarro del agua... La administración les ayuda en entubar o modernizar, porque les subvenciona» (Ignacio Trillo, ex delegado de Medio Ambiente en la Junta de Andalucía, Abril 28, 2017).

En 2010-2011 comenzaron los primeros diálogos en la comunidad de regantes para modernizar sus regadíos, inicialmente impulsados por la Junta de Andalucía y luego liderados por un grupo de pequeños empresarios y agro-productores del río Grande, en compañía de un ingeniero, experto en la modernización de regadíos. Ellos organizaron y convencieron a

5 Ver: http://www.europapress.es/andalucia/malaga-00356/noticia-fiscal-denuncia-regantes-rio-grande-captaragua-cumplir-condiciones-requeridas-20100613120038.html. 
los demás miembros de la comunidad de agricultores del río Grande para asumir los costes de la modernización.

Estos cambios han conllevado la creación de nuevas autoridades que ahora comienzan a ejercer control técnico, jurídico y financiero, definiendo así nuevos órdenes en los derechos de agua de los regantes. Uno de estos, tiene que ver con los derechos al acceso al agua del río: la comunidad de regantes ha dejado de construir sus pequeñas presas (azudes) y ahora realiza sus captaciones de agua a través del bombeo de aguas subterráneas. Desde allí el agua se transfiere por tubería y luego discurre sobre el trazado de las acequias. A través de contadores se le asigna a cada propietario un caudal determinado, que varía de acuerdo a la superficie de tierra que tenga.

Sin embargo, según los conocimientos locales de los moradores de río Grande, la modernización tendría efectos en la pérdida del acceso a otras fuentes locales de agua: pozos, aljibes y acuíferos. El agua que es conducida a través de las acequias para inundar las huertas por medio de los riegos a manta genera recargas hídricas en el subsuelo, alimentando acuíferos, pozos o aljibes de otras huertas y finalmente esa retorna nuevamente al río. Esta es una alternativa que contribuye en el autoabastecimiento de muchos agricultores para regar sus huertas o autoabastecer sus consumos domésticos. Abandonar los riegos a manta incidirá en la pérdida de la auto-recarga hídrica en el sistema del regadío del río Grande. ${ }^{6}$ Por estas razones, muchos moradores y agricultores consideran que los riegos a manta no son un «despilfarro» de agua, sino más bien se sienten enfrentados con un discurso modernizador poderoso que se encarga de marginar los regadíos tradicionales para justificar los riegos por goteo.

Estos factores han provocado que muchos agricultores se resistan a entrar en el proceso de modernización, que ha causado un debilitamiento en la auto-gobernanza local de su agua. En el momento que las acequias son entubadas, el reparto del agua deja de ser un asunto colectivo, y se convierte en un asunto individualizado, controlado por unos pocos que bajo el conocimiento de artefactos tecnológicos logran controlar la distribución del agua. Por otro lado, la modernización no es una alternativa rentable para la mayoría de los agricultores de río Grande, por los bajos precios con los que se comercializan los productos agrícolas, los elevados costos energéticos que se requieren para extraer agua y la poca extensión que tienen las fincas, la mayoría cultivadas para fines de autoabastecimiento. Estas condiciones no permiten que los agricultores tengan capacidad financiera para tecnificar sus sistemas de riego sin asumir un alto riesgo. El proceso de modernización, a medio plazo, tiende a facilitar la transferencia del control de las nuevas infraestructuras hidráulicas a la Junta de Andalucía y los pequeños empresarios agrícolas que lideran este proceso; ellos pasarían a controlar el volumen del consumo de agua.

Sin embargo, los regantes que decidieron entrar en el proceso de modernización (aproximadamente un $30 \%$ del total de la comunidad) sostienen que fue la única alternativa que

6 Actualmente no se cuenta con estudios cuantitativos. 
encontraron para no perder sus derechos de agua sobre el río - y sobre todo poder seguir cultivando, mantener la huerta y legarla a sus hijos en las mejores condiciones. También sostienen que entrar en el proceso de modernización les garantizaría mantener su derecho de agua comunitario, porque demostraban que se adaptaban a las normas ambientales orientadas a la economía del agua y protección del ecosistema. Dicen que les daría garantía jurídica, y por ende seguridad hídrica frente a una nueva intención de transferir aguas del río Grande hacia Málaga. Así mismo, sus tradicionales prácticas de riego, la construcción de la presa para captar aguas del río y sus riegos a manta, dejarían de ser denunciados por parte de las autoridades ambientales.

Sin embargo, de acuerdo con expertos académicos y muchos pobladores (entrevistas 2016-2017), la modernización tiene como objetivo liberar caudales para transferirlos hacia los lugares donde «se concentra la demanda». Al ahorrar y evitar al máximo la «pérdida de agua» dentro del sistema de riego se asegura que esta quede disponible para la industria turística y urbana concentrada en el litoral mediterráneo. En palabras de un académico (marzo 24, 2017): «Por ello en Andalucía se siguen promoviendo ese tipo de infraestructuras para darle el máximo uso a los recursos disponibles. La intención de mejorar, no es para el medio ambiente, ni recuperar el caudal fluvial o seguir aplicando la Directiva Marco del Agua... sino que responden a intereses del modelo antiguo, poner en producción cuantas más tierras hayan».

La modernización de los regadíos continúa sosteniendo el mismo modelo de la gestión de oferta que se ha venido desarrollando con la construcción de las grandes obras hidráulicas. Ha sido una estrategia socialmente aceptada al conciliar la noción del «ahorro máximo del agua» bajo dos posiciones sociales: proteger caudales ecológicos y asignarla a los lugares donde se concentra la población. Sin embargo, sus efectos son contradictorios, las extracciones de aguas subterráneas se están multiplicando, escapándose del control colectivo e incluso oficial. Mientras tanto, el continuo crecimiento urbano y de la industria turística sigue aumentado las presiones hídricas sobre el río Grande. Es el caso de los municipios cercanos a la Costa de Sol, como Cártama, Alhaurín de la Torre, Pizarra y Coín.

\section{LOS RETOS DE LA MOVILIZACIÓN SOCIAL}

Finalizando 2016, exdirigentes de la Confederación Hidrográfica del Sur, la Agencia Andaluza del Agua y actuales funcionarios de la Junta de Andalucía, anunciaron en diferentes periódicos la necesidad de retomar la construcción de la presa de Cerro Blanco en el río Grande. ${ }^{7}$ Diversos argumentos e intereses emergen para justificar nuevamente su construcción. El primero está centrado en la urgencia de controlar y regular flujos de agua para evitar

7 Tomado de: http://www.diariosur.es/malaga-capital/201612/10/junta-propone-retomar-azud-20161210011243-v. html. 
inundaciones, argumento que se fortaleció y coincidió con las fuertes lluvias que inundaron a Málaga en diciembre del 2016. El segundo tiene que ver con dar continuidad a los proyectos de urbanización de los municipios vecinos, los cuales se paralizaron por no tener concesiones de agua y fueron cerrados al no garantizar el abastecimiento hídrico. El tercero está asociado a la presión que ejercen los agro-empresarios de la Axarquía, comarca dedicada a la producción intensiva de cultivos tropicales y que por la sobreexplotación de sus acuíferos requiere con urgencia usar aguas de otra presa que abastece a Málaga, la Viñuela. Para ellos la construcción de la presa del río Grande posibilitaría usar libremente las aguas de la Viñuela. Y por último, a EMASA le interesa acceder a las aguas de río Grande por la gran calidad que tienen en la parte alta y por el ahorro en costes energéticos. El agua que se envía a Málaga debe bombearse, mientras que la del río Grande bajaría por gravedad y ampliaría la capacidad de acción, al cubrir las futuras demandas hídricas de las construcciones residenciales que quedaron paralizadas durante la crisis económica.

Estas alarmas han reactivado la movilización de los miembros y líderes de la Coordinadora en Defensa del río Grande. La Coordinadora reclama que antes de seguir pensando en la regulación de las aguas de río Grande, se invierta en tecnologías para la purificación y reciclaje de aguas residuales por cada área urbana de la subcuenca del río Grande, proyectos cuyo financiación ya han sido otorgada por la UE pero que no se han ejecutado por negligencia política. ${ }^{8}$ Por otro lado, el movimiento social declara que las inundaciones sucedidas en los últimos meses del 2016 no están relacionadas con la falta de una presa, sino que precisamente son producto de las irregularidades que provienen de la misma planificación urbana: los lugares que se inundaron coinciden con las áreas de humedales y antiguos cauces fluviales, zonas que antiguamente han pertenecido al río.

Por su parte, los agricultores que han entrado en el proceso de la modernización de regadíos, especialmente los líderes que la han impulsado, evitan discutir en sus reuniones la posibilidad de construcción de la presa. Esto agudizaría los conflictos entre los regantes y temen que muchos desistan o se retiren del proceso. Por ahora, lo que han acordado, es que si entran en la lucha contra la presa se hará a nombre individual, pero no en nombre de la comunidad de los regantes, pues temen que la Junta de Andalucia vuelva a tomar represalias contra ellos, tales como restricciones en los volúmenes de agua adjudicados, especialmente cuando están invirtiendo monetariamente para defender sus derechos de agua. El tránsito hacia la modernización de los regadíos está generando divisiones entre la comunidad de los agricultores y un debilitamiento en la lucha colectiva para proteger las aguas de río Grande. Especialmente, por las fisuras que se están presentando en el manejo comunitario de las aguas. Con la expansión de los riegos por goteo, la amenaza ya no es externa, sino interna: ahora todos buscan acceder a las aguas subterráneas, se está perdiendo el control colectivo

8 Ver: https://www.aguasresiduales.info/revista/noticias/ralentizadas-las-obras-de-depuracion-de-las-aguas-quese-vierten-al-guadalhorce-en-ma-2qfWV 
de los sistemas, y grupos de regantes están enfrentados entre sí por defender sus derechos tradicionales de agua frente a las amenazas modernas.

Desde otra perspectiva, para muchos habitantes, como los jóvenes agricultores de Coín, la lucha y la defensa del río Grande debe estar centrada en revivir la agricultura bajo un modelo alternativo que esté basado en los principios de la agro-ecología y el apoyo del autoconsumo local. Y muchos de los agricultores que se modernizan sostienen que la lucha en río Grande continua a través del mantenimiento de la actividad agraria local mediante la transformación hacia un sistema híbrido, en donde acequias históricas se combinan con tubos y motobombas nuevas. La lucha por el agua en río Grande transita entre la resistencia a abandonar aquel pasado, que actualmente sigue garantizando la libertad en el acceso y distribución colectiva de sus aguas, y la búsqueda de alternativas que aseguren sus derechos de agua por la vía legal, adaptándose a las normativas nacionales y ambientales a través del proceso de modernización.

\section{DisCUSIONES Y CONCLUSIONES}

Este articulo ha analizado como el discurso modernizador y sus verdades técnico-políticas objetivistas y universalizantes han sido contestadas, de acuerdo con la capacidad que han tenido los actores locales de movilizarse, influir y transformar las relaciones socio-políticas (Hoogesteger et al., 2016; Romano, 2017). El caso ilustra como los dos proyectos de modernización hidráulica en río Grande se expanden a costa de las realidades vernaculares. Al mismo tiempo, las presiones por el agua se intensifican, tanto en el área rural por la ampliación de áreas bajo riego tecnificado, como en el área urbana respondiendo al descontrol en el crecimiento de la industria turística y urbana. La modernidad intensifica y construye situaciones de escasez hídrica (véase también Aguilera-Klink et al., 2000; Hommes y Boelens, 2017; Mehta, 2006; Mena-Vásconez et al., 2017), en las que paradójicamente la construcción de nuevas obras hidráulicas o la tecnología eficiente para ahorro de agua se siguen presentando como soluciones ambientales y óptimas para enfrentar las incertidumbres del cambio climático (Boelens y Vos, 2012; Sanchis-Ibor et al., 2017).

En respuesta a esta crisis, otros conocimientos emergen a través de las movilizaciones sociales de las plataformas antiembalses desde ámbitos nacionales y locales. Estas se unen para rechazar la continuidad del manejo tecnocrático de los ríos que, en lugar de resolver la crisis de la gestión del agua, la agudizan por la misma monopolización del poder en el uso y control de las obras hidráulicas. Estas movilizaciones son apoyadas por redes de académicos e intelectuales de distintas partes del país. De esta manera, el conocimiento experto científico no solo es movilizado para defender intereses de sectores poderosos sino también puede fortalecer (y fortalecerse) las experiencias y conocimientos de los movimientos antiembalses. Las contestaciones epistemológicas posicionan y relacionan a actores diversos, quienes adquieren las redes, información y capacidad para defender sus batallas hídricas. 
Este artículo también ilustra como las entidades oficiales cambian y adecuan sus discursos y propuestas de desarrollo hidráulico a medida que las contestaciones evolucionan y se fortalecen. El anuncio de la construcción de un azud y un canal de conducción alternativos a la presa buscó estratégicamente apaciguar las protestas de los agricultores mayores, pero acentuó la movilización de la población más joven, la asociación Jara. Esta organización apoyó la defensa del río Grande visibilizando los conocimientos ecológicos del río y destacando la importancia de mantenerlo vivo y libre de presas. Su movilización se caracterizó por conectar actores heterogéneos, facilitando su expansión hacia diversas escalas e integrando las diversas formas de conocimiento, desde saberes populares a los científicos.

El artículo demuestra también como el movimiento ha tenido un efecto catalizador para unificar múltiples actores y diversas escalas cuando la amenaza fue externa y visible, pero se complicó cuando esta se enmascaró sutilmente como proyecto comunitario «desde abajo» y «participativo». Paradójicamente, la ideología que sustentó la lucha social en río Grande - la protección del río vivo y libre - se convierte en la estrategia que usan entidades oficiales y ciertos grupos ambientalistas para restringir y alterar los derechos consuetudinarios de agua de las comunidades de regantes. Las contestaciones frente a este fenómeno dividen a la comunidad y complican la lucha unificada contra las transferencias de las aguas rurales a la ciudad.

Argumentamos que los retos de los movimientos sociales deben ir más allá de la simple oposición a la construcción de la presa o defensa del río: deben estar centrados en reivindicar sus derechos de usos y manejo del agua, y así generar procesos de apropiación colectiva para la defensa del río como un bien ambiental que asegure la supervivencia de su legado y prácticas culturales. La lucha por el río Grande plantea el reto de repensar la gestión del agua desde conceptos menos universalistas y homogeneizantes para dar lugar a una gestión hídrica más diversificada, en donde los crecimientos de ciertos sectores económicos sean acordes a sus capacidades territoriales y no comprometan el bienestar social de otras zonas, marginando las comunidades rurales.

\section{Agradecimientos}

Las bases empíricas de este articulo fueron posibles gracias al constante apoyo de la asociación Jara, especialmente Salvador Sánchez. Agradecemos también a Juan González, Pepe Cortés, Pepe Urbano, Juan Marmolejo, Cristóbal y Sebastián Hevilla, y todos los demás regantes, agricultores, habitantes de Coín por el tiempo dedicado durante la investigación. De igual manera, agradecemos a los (ex) funcionarios de la Junta de Andalucía, de EMASA por abrirnos las puertas y atender a todas nuestras entrevistas. 


\section{REFERENCIAS}

Aguilera-Klink, F., E. Pérez-Moriana y J. Sánchez-García (2000): The social construction of scarcity. The case of water in Tenerife (Canary Islands). Ecological Economics, 34(2), 233-245.

Arce, A. y Long, N. (1987): The dynamics of knowledge interfaces between Mexican agricultural bureaucrats and peasants: a case study from Jalisco. Boletín de Estudios Latinoamericanos y del Caribe, (43), 5-30.

Bebbington, A. (1996): Movements, modernizations, and markets. In Liberation ecologies: Environment, development, social movements, Peet, R y Watts, M. (Eds.), pp. 86-109. Routledge: London.

Boelens, R. (2015): Water, Power and Identity. The cultural politics of water in the Andes. Routledge, London y New York.

Boelens, R. y Vos, J. (2012): The danger of naturalizing water policy concepts: water productivity and efficiency discourses from field irrigation to virtual water trade. Agricultural Water Management, 108, 16-26, doi:10.1016/j.agwat.2011.06.013.

Boelens, R. y Post Uiterweer, N.C. (2013): Hydraulic heroes: The ironies of utopian hydraulism and its politics of autonomy in the Guadalhorce Valley, Spain. Journal of Historical Geography, 41, 44-58, doi:10.1016/j.jhg.2012.12.005.

Copitzy, A (2008): Territorio y resistencia Social. La lucha en contra de la construcción de las presas y trasvases (Aragón, 1985-2010). Zapopam, Jal. El Colegio de Jalisco, México

Corominas, J. y Cuevas-Navas, R. (2017): Análisis crítico de la modernización de regadíos. Pensando el futuro: ¿cómo será el nuevo paradigma? en Berbel, J. y Gutiérrez-Martín, C. (eds.): Efectos de la modernización de regadíos en España. Cajamar Caja Rural, Almería.

Costa, J. (1911): Política hidráulica: misión social de los riegos en España. Vol. 2. Biblioteca J. Costa, Madrid.

Delgado, M. y L. Del Moral (2016): Los megaproyectos en Andalucía. Relaciones de poder y apropiación de riqueza. Aconcagua, Sevilla

Del Moral, L. y D. Saurí (1999): Changing Course: Water Policy in Spain. Environment: Science and Policy for Sustainable Development, 41(6), 12-36.

Duarte-Abadía, B. y Boelens, R. (2016): Disputes over territorial boundaries and diverging valuation languages: The Santurban hydrosocial highlands territory in Colombia. Water International, 41(1), 15-36, doi:10.1080/02508060.2016.1117271.

Duarte-Abadía, B. y R. Boelens (2019): Colonizing Rural Waters. The politics of hydro-territorial transformation in the Guadalhorce Valley, Málaga, Spain. Water International, publ. próx.

Duarte-Abadía, B., Boelens, R. y Roa-Avendaño, T. (2016): Hydropower, encroachment and the re-patterning of hydrosocial territory: The case of Hidrosogamoso in Colombia. Human Organization, 74(3), 243-254, doi:10.17730/0018-7259-74.3.243.

Estevan, A.E:. Herencias y problemas de la política hidráulica española. Bakeaz, Bilbao.

Fernández Clemente, E. (2000): De la utopía de Joaquín Costa a la intervención del estado: un siglo de obras hidráulicas en España. Universidad de Zaragoza, Zaragoza. 
Foucault, M. (1980): Power/knowledge. In C. Gordon (Ed.): Power/Knowledge: Selected interviews and other writings 1972 - 1978. Pantheon Books, New York.

GajIC, T. (2007): Fronteras líquidas: Agua y bio-política de la territorialidad en España. Arizona Journal of Hispanic Cultural Studies, 11(1), 25-41.

Gómez, M.L. (1998): El Genal apresado: agua y planificación: ¿desarrollo sostenible o crecimiento ilimitado?. Bakeaz, Bilbao.

Hommes, L. y Boelens, R. (2017): Urbanizing rural waters: Rural-urban water transfers and the reconfiguration of hydrosocial territories in Lima. Political Geography, 57, 71-80, doi: 10.1016/j.polgeo.2016.12.002.

Hommes, L. y Boelens, R. (2018): From natural flow to «working river»: hydropower development, modernity and socio-territorial transformations in Lima's Rímac watershed. Journal of Historical Geography, 62, 85-95, doi: 10.1016/j.jhg.2018.04.001.

Jiménez SÁnchez, M., y Poma, A. (2011): Lógicas en conflicto. Conocimiento experto y política en la movilización social en defensa de río Grande (Málaga). Arxius, 25(11), 59-70.

Hoogesteger, J., R. Boelens y M. Baud (2016): Territorial pluralism: water users' multi-scalar struggles against state ordering in Ecuador's highlands. Water International, 41(1), 91-106, doi: 10.1080/02508060.2016.1130910.

Illich, I. (1980): Vernacular values. Philosophica, 26, 2-32.

KaIKA, M. (2006): Dams as symbols of modernization: The urbanization of nature between geographical imagination and materiality. Annals of the Association of American Geographers, 96(2), 276-301.

Long, N. (2003): Development sociology: actor perspectives. Routledge.

López-Gunn, E., Zorrilla, P., Prieto, F. y Llamas, R. (2012): Lost in translation? Water efficiency in Spanish agriculture. Agric. Water Management, 108, 83-95.

Mena-Vásconez, P., Boelens, R. y Vos, J. (2016): Food or flowers? Contested transformations of community food security and water use priorities under new legal and market regimes in Ecuador's highlands. Journal of Rural Studies, 44, 227-238, doi: 10.1016/j.jrurstud.2016.02.011.

Menta, L. (2006): Whose scarcity? Whose property? The case of water in western India. Land Use Policy, 24(4), 654-663.

Nixon, R. (2010): Unimagined communities: developmental refugees, megadams and monumental modernity. New Formations, 69(69), 62-80.

Ortí, A., (1984): Política hidráulica y cuestión social: orígenes, etapas y significados del regeneracionismo de Joaquín Costa. Agricultura y Sociedad, 32, 11-107.

Poma, A. y T. Gravante (2015): Analyzing Resistance from below: A Proposal of Analysis Based on Three Struggles against Dams in Spain and Mexico: Capitalism Nature Socialism, 26(1), 59-76.

PRÉ, L. DU (2017): Río Grande's troubled water. The struggles between rural water users and external actor alliances over the materialization of the rural hydrosocial territory. Wageningen University, Wageningen.

Puche, F. (2003): Río Grande: cuaderno de trabajo por la nueva cultura del agua. Mesa de Amig@s de los Ríos, Málaga. 
Pfaffenberger, B. (1992): Technological dramas. Science, Technology, Ë Human Values, 17(3), 282312.

Romano, S.T. (2017): Building Capacities for Sustainable Water Governance at the Grassroots: «Organic Empowerment» and Its Policy Implications in Nicaragua. Society $\mathcal{E}$ Natural Resources, 30:4, 471-487.

Sampedro, D. y Del Moral, L. (2014): Tres décadas de política de aguas en Andalucía. Cuadernos Geográficos, 53(1), 36-67.

Sanchis-Ibor, C., García-Mollá y M., Avellà, L. (2016): Efectos de la modernización del regadío en las entidades de riego de la Comunidad Valenciana. Boletín de la Asociación de Geógrafos Españoles, 72, 9-36.

Sanchis-Ibor, C., Boelens, R. y García-Mollá, M. (2017): Collective irrigation reloaded. Recollection and re-moralization of water management after privatization in Spain. Geoforum, 87, 38-47.

Stuiver, M., Leeuwis, C. y van der Ploeg, J. D. (2004): The power of experience: farmer's knowledge and sustainable innovations in agriculture. Wiskerke, H. and J.D. van der Ploeg, Seeds of Transitions, 93-118. Assen, Van Gorcum.

Swyngedouw, E. (1999): Modernity and Hybridity: Nature, Regeneracionismo, and the Production of the Spanish Waterscape, 1890-1930. Annals of the Association of American Geographers, 89, 3, 443-465.

Swyngedouw, E. (2007): Tecnonatural revolutions: the scalar politics of Franco's hydro-social dream for Spain, 1939-1975. Trans Inst Br Geogr. 32, 9-28.

Swyngedouw, E. (2015): Liquid Power: Contested Hydro-Modernities in 20th Century Spain. MIT Press, Cambridge.

Swyngedouw, E. y Boelens, R. (2018): «... And Not A Single Injustice Remains»: Hydro-Territorial Colonization And Techno-Political Transformations In Spain. En: R. Boelens, T. Perrault y J. Vos (eds.): Water Justice, pp. 115-133. Cambridge University Press, Cambridge.

Cómo citar este artículo:

Duarte Abadía, B., Boelens, R. y Du Pré, L. (2018). Alianzas antiembalse

y luchas hidroepistemológicas. Los retos de la gestión colectiva del agua en el río Grande,

Málaga, España. Cuadernos de Geografía, 101, 107-126.

https://doi.org/10.7203/CGUV.101.13723

\section{(C) $(\Theta \Theta \Theta$}

Este obra está bajo una licencia de Creative Commons Reconocimiento-NoComercial-SinObraDerivada 4.0 Internacional. 
